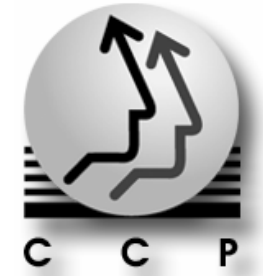

Población y Salud en Mesoamérica

Revista electrónica publicada por el

Centro Centroamericano de Población

Universidad de Costa Rica, 2060 San José, Costa Rica

http://ccp.ucr.ac.cr

Población y Salud en Mesoamérica

Revista electrónica

Volumen 3, número 1, artículo 3

Julio - diciembre, 2005

Publicado 1 de julio, 2005

http://ccp.ucr.ac.cr/revista/

\title{
Tamaño de familia deseado: análisis basado en la Encuesta Nacional de Salud Reproductiva y Migración, Costa Rica 1999.
}

\section{Ericka Méndez Chacón}

(C) 2005 Centro Centroamericano de Población 


\title{
Tamaño de familia deseado: análisis basado en la Encuesta Nacional de Salud Reproductiva y Migración, Costa Rica 1999.
}

\author{
Ericka Méndez Chacón ${ }^{1}$
}

\begin{abstract}
RESUMEN
La fecundidad es un componente del cambio demográfico, ya que su aumento o disminución incide en el tamaño y estructura de una población. En las encuestas de fecundidad se incluyen preguntas que pretenden medir la demanda de niños o nacimientos que una mujer desearía tener al momento de la encuesta. Los nacimientos no deseados o una desigualad entre el numero de hijos tenidos y los hijos deseados se puede medir sustrayendo ambas respuestas; de esta manera se cuenta con las mujeres quienes manifestaron tener al momento de la encuesta un número mayor de nacimientos al la cantidad de hijos deseados. Se encontró que la probabilidad de tener más hijos de los deseados aumenta con la edad de la madre y la asistencia a la iglesia, mientras que la mayor escolaridad y el estar unida o casada disminuye la probabilidad.
\end{abstract}

\section{INTRODUCCION}

La fecundidad no deseada puede ocurrir por dos razones: por falla en los métodos anticonceptivos utilizados o por demanda insatisfecha (deficiencia de oferta de métodos) (Bermúdez y Rosero 1993). Puede ser no deseada en el momento del embarazo (embarazos no planeados), ya que se deseaba un espaciamiento, lo que se conoce como fecundidad a destiempo, o puede ser no deseada del todo (González y Chen, 1999).

El estudio de la fecundidad no deseada se ha cuantificado utilizando el indicador "Tasa global de fecundidad deseada”, (TGFD) ${ }^{2}$ la cual es un indicador del número de hijos deseados tomando en cuenta, el tamaño de familia ideal y el número de hijos sobrevivientes, así como los años persona de exposición. Refleja lo que sería la tasa global de fecundidad, es decir, los hijos que tendría una mujer al final de su vida fértil si tiene los hijos de acuerdo a las tasas de fecundidad por edad observadas en ese periodo y sin estar expuesta a riesgos de mortalidad, si solo ocurrieran nacimientos deseados.

La comparación de esta tasa con la global da un indicador de la fecundidad no deseada (TFND), esto es:

\section{TFND $=$ TGF - TGFD}

\footnotetext{
${ }^{1}$ Investigadora, Proyecto “Costa Rica: longevidad y envejecimiento saludable” (CRELES-CCP), Universidad de Costa Rica, ericka@ccp.ucr.ac.cr

2 http://ccp.ucr.ac.cr/cursoweb/3212wtgf.htm
} 
Esto es de utilidad para formular políticas de planificación familiar (demanda de limitación y demanda de espaciamiento) y evaluar un descenso de la fecundidad en el futuro.

Bongaarts (1997) menciona que la teoría convencional de transición de la fecundidad predice que al estar un país en desarrollo, los costos de crianza de los hijos se elevan y los beneficios que se pueden obtener de estos caen, lo que lleva a las parejas a querer familias pequeñas. Esto lo nota al comparar la fecundidad deseada de los países del África sub-Sahara donde la tasa más baja observada 5.8 con los de América Latina y el Caribe en donde la tasa más alta observada es 4.5.

Costa Rica no escapa a esta realidad, las encuestas muestran que el número medio de hijos deseados pasó de 4.8 en 1976 a 2.7 en 1999 (González y Chen, 1999).

Bermúdez y Rosero (1993) encuentran que entre las mujeres en edad de 15-49 años, un 22\% de la fecundidad fue no deseada y que esta se incrementa con la edad de la mujer. Encontraron además, que para 1993 el último nacimiento no deseado se debió a un deseo de cesar la procreación más que a espaciarla como había sucedido en encuestas anteriores. Existen diferencias en este deseo en el aumento en el número de hijos vivos, la edad de la mujer (principalmente las jóvenes), los años de educación y la asistencia a la iglesia.

Como parte de los resultados obtenidos en la Encuesta de Salud Reproductiva de 1999, González y Chen encuentran que con respecto al deseo de quedar embarazada en ese momento, no embarazarse o esperar un tiempo antes del nacimiento del último hijo está asociada las variables: número de hijos vivos, años de estudio de la entrevistada, ser católica o no y la condición de trabajo.

En 1990 la Asociación Demográfica Costarricense realizó un estudio sobre el embarazo no deseado en las mujeres en gestación que asistieron a la Consulta de Control Prenatal en clínicas y hospitales públicos. En este estudio el término embarazo no deseado "mide la percepción inicial que tuvo la mujer de su embarazo como inaceptable, inconveniente o fuente de conflictos internos o externos. Mide la reacción inmediata de insatisfacción o inapetencia que experimentó la mujer al saber de su estado”. (Madrigal, 1992).

Algunos resultados encontrados indican que el embarazo no deseado se presenta en mayor magnitud en las mujeres adolescentes y aquellas con edad igual o superior a 40 años, las que tienen 6 hijos o más, las que están desunidas o solteras, mujeres con bajo nivel educativo y las que tienen bajo nivel de ingreso. En este mismo estudio se plantea el término de Embarazo no Planeado, el cual se define como "Aquel embarazo en el que no media una meditación detenida y se produce por accidente, sorpresa o descuido o en un momento inesperado” (Madrigal, 1992).

Se encontró que la no planeación está ligada a los embarazos no deseados, sin embargo existen excepciones y hay embarazos no planeados que sí son deseados. Al respecto se encontró que las variables antes mencionadas son también determinantes en los embarazos no planeados. 
Planteados estos dos enfoques, surge la inquietud de si realmente la diferencia de los hijos tenidos y el tamaño ideal de familia da como resultado hijos no deseados, para efecto de este estudio, se utilizará esta diferencia como una aproximación a este hecho, tomando en cuenta que en algún momento un hijo no planeado pudo causar cierta inquietud a una mujer e incluso deseo de no tenerlo aunque fuera por un corto espacio de tiempo.

\section{DATOS Y METODOS}

Para la construcción del modelo se tomaron los datos de la Encuesta Nacional de Salud Reproductiva y Migración 1999. La población de estudio se refiere a aquellas mujeres entre 18 y 44 años de edad que estaban por primera vez embarazadas o tenían uno o más hijos vivios al momento de la encuesta.

Se eliminaron aquellos casos cuya respuesta a la pregunta ¿Cuántos serían los hijos que le gustaría tener en toda su vida? fue un valor perdido o ignorado o tienen el código de otra respuesta.

Se utilizó un modelo de regresión logística para describir la relación existente entre ciertas variables con aquella que indica si la mujer había tenido al menos un hijo más al número de familia deseado. La definición de las variables se realizó de la siguiente manera:

Variable Respuesta:

Hijos no deseados: Variable discreta (0-1). Se obtuvo a través de la diferencia entre el número de hijos tenidos vivos y el número de hijos deseados.

Se tomó como 0 hijos no deseados aquella diferencia que dio menor o igual a cero ya que las mujeres querían tener igual o más hijos de los que en ese momento tenían y como 1 los que dieron exactamente uno o más hijos, como indicador de haber completado su tamaño de familia deseado y tener en ese momento más hijos.

Para el caso de las mujeres que estaban embarazadas en ese momento y además era su primer embarazo se contabilizó como un hijo no deseado las que respondieron que quería esperar o no querían el embarazo a la pregunta “¿Quería usted quedar embarazada en ese momento, le hubiera gustado esperar un poco o hubiera preferido no quedar embarazada más?”.

Para realizar la resta, se agregó a la cantidad de hijos tenidos vivos, un hijo para las mujeres embarazadas que ya tenía al menos un hijo vivo.

Variables explicativas:

Zona de residencia: es una variable indicadora (0-1) de la zona de residencia en urbano (1) y rural (0)

Edad: Variable continua. Edad de la mujer en años cumplidos. 
Educación: Variable continua. Se refiere a los años de educación (completos en el sistema educativo formal) de la mujer.

Asistencia a la iglesia: Se refiere a la frecuencia con que asisten a la iglesia. Se crearon cuatro variables:

1. Al menos una vez por semana.

2. Una, dos o tres veces al mes.

3. Menos de una vez por mes.

4. Nunca.

Cada una de estas es una variable indicatríz (0-1), se tomó como referencia “Al menos una vez por semana”.

Condición laboral: Trabaja (1) o no trabaja (0)

Dentro de las mujeres que trabajan se consideró también aquellas que a pesar de decir que solo estudiaban o no trabajaban respondieron afirmativamente a la pregunta: "Como usted sabe, algunas mujeres se ganan algo vendiendo, cosiendo ajeno, otras ayudan en la finca o negocio de la familia, haciendo cosas similares. ¿Hace usted alguna de estas cosas o algo parecido?”.

\section{RESULTADOS}

Cerca de un 22\% de las mujeres embarazadas o con al menos un hijo nacido vivo tiene uno o más hijos no deseados, de acuerdo a la definición antes mencionada. El promedio de hijos tenidos vivos es 2.6. Un 18\% de ellas manifestó no estar unida o casada.

El 49\% de las mujeres tiene algún grado de secundaria aprobado. El 47\% trabaja, el 76\% de las mujeres manifestaron ser católicas y en general la asistencia a la iglesia es bastante frecuente (51\% asiste al menos una vez por semana). El 54\% de las mujeres viven en la zona rural y la mayoría son costarricenses por nacimiento (87\%).

En general se puede decir que han cuidado de su salud, el 85\% no ha fumado nunca, el 90\% se ha hecho un Papanicolau alguna vez en su vida y que gran proporción de ellas (77\%) se lo hizo en los años 98-99. El 76\% se ha vacunado contra el tétano en los últimos 10 años.

En relación con los métodos anticonceptivos todas la mujeres conocen de al menos un método y el 95\% los han usado. La mayoría de las mujeres comenzaron a usarlos en edades de 18 a 20 años. El 55\% ellas tenía uno o más hijos cuando comenzó a usarlos.

La mayoría -más de un 90\%- está de acuerdo en que se dé clases de educación sexual en los colegios, han asistido a la consulta de planificación de la Caja Costarricense del Seguro Social (CCSS) y están a favor de la planificación. 
Otro aspecto importante relacionado con el tema es el aborto. Al respecto el porcentaje de mujeres que está de acuerdo con este cuando peligra la vida de la madre es del 39\%, el 30\% lo apoya cuando peligra la salud de la madre, el $20 \%$ cuando la mujer fue embarazada por el padre o un hermano, el 12\% cuando el niño será anormal o el embarazo fue producto de una violación. Es de interés mencionar que solo cerca del $2 \%$ apoya el aborto cuando el hombre no desea casarse, no pueden mantener los hijos o cualquier otro motivo.

El cuadro 1 muestra los resultados del ajuste del modelo con las variables antes indicadas, se encontró que la edad, los años de estudio, la asistencia a la iglesia y la condición de unión son factores que están asociados al hecho de tener hijos no deseados.

En el cuadro 2 se presentan las razones de ventaja las cuales permiten concluir que: en relación a la edad de la mujer, la razón de ventaja de tener uno o más hijos no deseados es 1.09 con respecto a las que tienen un año menos de edad. Existe menor probabilidad de tener algún hijo no deseado en las mujeres que tienen más años de estudio. Las mujeres que asisten a la iglesia una, dos o tres veces al mes presentan una razón de ventaja de 2.5 con respecto a las que asisten al menos una vez por semana. Esta razón es apenas menor cuando se trata de mujeres que no asisten a la iglesia (2.25). Los odds de tener un hijo no deseado entre las mujeres casadas o unidas son 0.56 con respecto a las no unidas.

En cuanto al ajuste del modelo, este presenta un valor del Pseudo $\mathrm{R}^{2}$ de $10.34 \%$ (es decir, este modelo explica un $10.34 \%$ más que el modelo nulo), esto permite concluir que el modelo es adecuado con respecto al modelo nulo, adicionalmente no parece haber evidencia de necesitar más información (Prob>chi2 $=0.4043$ ) con respecto a un modelo saturado. La prueba de Hosmer y Lemeshow indica que no existe evidencia estadística para rechazar el modelo ajustado ( $\mathrm{P}>$ chi $2=0.5075)$, es decir, el ajuste es bueno.

El modelo clasifica correctamente el 78\% de las observaciones, la sensibilidad ${ }^{3}$ alcanzada es de $11 \%$ y la especificidad ${ }^{4}$ de $97 \%$. El área bajo la curva ROC es de 0.7196 lo que indica una adecuada discriminación. La probabilidad que maximiza la sensibilidad y la especificidad en este modelo es aproximadamente 0.20. La especificidad y sensibilidad nuevas tomando como punto de corte este valor son $71 \%$ y $61 \%$ respectivamente.

\section{DISCUSION}

Se esperaba que en este modelo, haciendo la similitud con la teoría de transición de la fecundidad mencionada por Bongaarts, la zona de residencia al interior de Costa Rica resultara significativa. El modelo muestra que no existe diferencia entre la probabilidad de tener hijos no deseados en la zona urbana con respecto a la rural.

Se comprueba que la edad, como en estudios anteriores (Bermúdez y Rosero 1999), es influyente en el hecho de tener hijos no deseados. Así cuando existen diferencias de 10 años

${ }^{3}$ Porcentaje real de casos de hijos no deseados clasificados correctamente por el modelo.

${ }^{4}$ Porcentaje real de casos de hijos deseados clasificados correctamente por el modelo. 
en la edad la razón de ventaja es de 2.36, es decir una mujer que sea 10 años mayor a otra con las mismas características, es más probable que tenga al menos un hijo no deseado.

Se evidencia que a más años de estudio, es menos probable no querer tener algún hijo, de esta manera, las mujeres que tienen 3 años más de educación presentan una razón de odds de 0.6578. Las mujeres que asisten poco o del todo no asisten a la iglesia es más probable que tengan un hijo no deseado.

Definitivamente el apoyo de la pareja parece ser importante en el deseo de tener un hijo. Es más probable un hijo no deseado cuando la mujer no está unida o casada que cuando si lo está.

Respecto a esta medición podría presentarse un sesgo debido a ciertos factores confusores que hacen que la cuantificación sea difícil. Entre estos factores se encuentran la mortalidad infantil que eventualmente llevaría a las mujeres a reponer algún hijo perdido, las preferencias de la composición familiar en cuanto a sexo y alguna limitación en la fecundidad o cambios en el espaciamiento de los nacimientos. Adicionalmente existe un proceso de racionalización en donde las mujeres adaptan el tamaño de familia deseado a la cantidad de hijos que ya han tenido.

Un aspecto importante, como se menciona en el informe de la Encuesta de Fecundidad de 1993, es que la pregunta ¿Cuántos serían los hijos que le gustaría tener en toda su vida?, la cual pone a la mujer en una situación hipotética y las respuestas dadas podrían ser el número de hijos que ya se tiene. De esta manera, un hijo puede ser, en cierto espacio de tiempo corto o largo, no deseado o no planeado, pero después ya no lo es. 


\section{REFERENCIAS}

Bermúdez Alicia y Rosero Luis (1993). Metas reproductivas y patrones de crianza de los hijos, Informe de trabajo No. 1

Bongaarts John (1997).Trends in unwanted childbearing in the developing world. Studies in Family Planning, 28, 4, 267-277.

Bongaarts John (1990).The Measurement of wnted Fertility. Population and Development Review, 16, 3, 487-506.

Caja Costarricense de Seguro Social (1993). Encuesta Nacional de Salud Reproductiva.

Chen et al. (2000). Salud reproductiva y migración nicaragüense en Costa Rica 19992000.

Family Care International, El embarazo no deseado, http://www.familycareintl.org/esp/trabajo/trabajoglobal.htm

Profamilia Colombia, Derechos sexuales y reproductivos al alcance de todos, http://www.profamilia.org.co/profamilia/P4/PDFS/25_derechos-sexuales.pdf

Madrigal et al. (1992). Deseo y planeamiento del embarazo. El embarazo no deseado en Costa Rica. Informe de resultados. 
Cuadro 1. Estimación de los coeficientes, errores estándar, valores del estadístico z, valores p de dos colas e intervalos de confianza para los coeficientes.

\begin{tabular}{lrrrrrr}
\hline \multicolumn{1}{c}{ Variable } & Coeficiente & $\begin{array}{r}\text { Error } \\
\text { estándar }\end{array}$ & $\mathbf{Z}$ & $\mathbf{p}>|\mathbf{z}|$ & $\begin{array}{c}\text { Intervalos de confianza } \\
(\mathbf{9 5 \% )}\end{array}$ \\
\hline Edad & 0.0861 & 0.0136 & 6.3200 & 0.0000 & 0.0594 & 0.1128 \\
Años de estudio & -0.1395 & 0.0286 & -4.8900 & 0.0000 & -0.1955 & -0.0836 \\
Trabaja & -0.2240 & 0.1937 & -1.1600 & 0.2470 & -0.6037 & 0.1556 \\
asistig2* & 0.3193 & 0.2187 & 1.4600 & 0.1440 & -0.1094 & 0.7481 \\
asistig3** & 0.9119 & 0.3555 & 2.5700 & 0.0100 & 0.2151 & 1.6086 \\
asistig4*** & 0.8130 & 0.2583 & 3.1500 & 0.0020 & 0.3066 & 1.3194 \\
casada & -0.5629 & 0.2355 & -2.3900 & 0.0170 & -1.0245 & -0.1014 \\
urbano & 0.0367 & 0.1974 & 0.1900 & 0.8520 & -0.3501 & 0.4236 \\
Constante & -2.7930 & 0.5376 & -5.2000 & 0.0000 & -3.8467 & -1.7393 \\
\hline
\end{tabular}

* Asiste una, dos o tres veces a la iglesia

** Asiste menos de una vez por mes

***Nunca asiste a la iglesia

Cuadro 2. Estimación de los Odds Ratio para cada una de las variables significativas e intervalo de confianza.

\begin{tabular}{lccc}
\hline & \multicolumn{3}{c}{ Intervalos de confianza } \\
Variable & OR & $\mathbf{( 9 5 \% )}$ & \\
\hline Edad & 1.090 & 1.061 & 1.119 \\
años de estudio & 0.870 & 0.822 & 0.920 \\
asistig3** & 2.489 & 1.240 & 4.996 \\
asistig4*** & 2.255 & 1.359 & 3.741 \\
casada & 0.570 & 0.359 & 0.904 \\
\hline
\end{tabular}

** Asiste menos de una vez por mes

***Nunca asiste a la iglesia 